\title{
High-bandwidth nanopositioning via active control of system resonance
}

(C) The Author(s) 2021. This article is published with open access at link.springer.com and journal.hep.com.cn

\begin{abstract}
Typically, the achievable positioning bandwidth for piezo-actuated nanopositioners is severely limited by the first, lightly-damped resonance. To overcome this issue, a variety of open- and closed-loop control techniques that commonly combine damping and tracking actions, have been reported in literature. However, in almost all these cases, the achievable closed-loop bandwidth is still limited by the original open-loop resonant frequency of the respective positioning axis. Shifting this resonance to a higher frequency would undoubtedly result in a wider bandwidth. However, such a shift typically entails a major mechanical redesign of the nanopositioner. The integral resonant control (IRC) has been reported earlier to demonstrate the significant performance enhancement, robustness to parameter uncertainty, guaranteed stability and design flexibility it affords. To further exploit the IRC scheme's capabilities, this paper presents a method of actively shifting the resonant frequency of a nanopositioner's axis, thereby delivering a wider closedloop positioning bandwidth when controlled with the IRC scheme. The IRC damping control is augmented with a standard integral tracking controller to improve positioning accuracy. And both damping and tracking control parameters are analytically optimized to result in a Butterworth
\end{abstract}

Received August 13, 2020; accepted November 2, 2020

Linlin LI, Limin ZHU

State Key Laboratory of Mechanical System and Vibration, School of Mechanical Engineering, Shanghai Jiao Tong University, Shanghai 200240, China

Linlin LI

State Key Laboratory of Fluid Power and Mechatronic Systems, Zhejiang University, Hangzhou 310027, China

Sumeet S. APHALE ( $₫)$

The Centre for Applied Dynamics Research, School of Engineering, University of Aberdeen, Aberdeen AB24 3UE, UK

E-mail: s.aphale@abdn.ac.uk

Limin ZHU

The Shanghai Key Laboratory of Networked Manufacturing and Enterprise Information, Shanghai 200240, China
Filter mimicking pole-placement - maximally flat passband response. Experiments are conducted on a nanopositioner's axis with an open-loop resonance at $508 \mathrm{~Hz}$. It is shown that by employing the active resonance shifting, the closed-loop positioning bandwidth is increased from 73 to $576 \mathrm{~Hz}$. Consequently, the root-mean-square tracking errors for a $100 \mathrm{~Hz}$ triangular trajectory are reduced by $93 \%$.

Keywords nanopositioning stage, high-bandwidth, resonant mode control, tracking control, integral resonant control

\section{Introduction}

Since its invention in 1986, the Atomic Force Microscope (AFM) [1] has enabled the probing and manipulating as well as fabricating matter, at nanometer scales. Consequently, it has continued to facilitate huge developments in a wide range of science and technology areas [2-4]. The positioning capabilities of an AFM is predominantly governed by the nanopositioner, which is generally employed to position the test sample under the microcantilever $[5,6]$. Though piezoelectric nanopositioners (both tube- and platform-type) are popularly employed in AFMs due to their advantages of modularity, repeatability, mechanical simplicity, ease of operation, their lightlydamped resonance and piezoelectric-actuator induced hysteresis and creep effects leave a lot to their desire positioning performance [7-10]. The main effect of this lightly-damped resonance manifests in the unwanted oscillatory positioning errors when high-frequency components of the desired scanning trajectory excite the system resonance $[11,12]$. Typically, the triangular trajectories used in raster scanning of AFM contains oddharmonic high-frequency components, making it rather challenging for high-speed operations.

In the past decades, several methods have been reported to improve the tracking performance of the positioning 
stages [7,11,13-19]. These methods introduced to overcome the positioning limitation by the lightly-damped mechanical resonance take a very important position in the above methods, and can be roughly grouped into two categories: (i) Mechanical improvements that include development of new structures, like flexure-based mechanisms aimed at achieving high resonant frequencies $[13,20,21]$, and (ii) control strategies that include various feedforward and feedback strategies, such as notch filters $[22,23]$, positive position feedback control $[9,24]$, polynomial-based control [25], positive acceleration, velocity, and position feedback control [26], integral resonant control (IRC) [27-29], delay time control [15], force feedback control [30], and linear quadratic Gaussian controller [31], that impart significant damping to the resonant mode. These damping schemes are then augmented with suitably gained tracking controllers to overcome errors introduced by hysteresis and creep nonlinearities arising from the nonlinear dynamics of the piezoelectric actuators employed $[9,15,26]$. In most reported implementations, the damping control and tracking control designs are sequentially performed, whereby the tracking control loop invariably changes the location of the damped system poles, consequently narrowing the achievable positioning bandwidth [32]. To overcome this and to ensure a maximally flat-band closed-loop response, a simultaneous controller design mimicking the Butterworth pole-placement pattern was proposed in Ref. [33]. Although significant improvements have been achieved by the techniques mentioned above, the achievable closed-loop bandwidth is still limited by the original open-loop resonance frequency of the respective positioning axis.

In order to achieve a wider bandwidth without re-design of the mechanical structure, this paper presents an active control method to shift the resonance to a higher frequency. To further enhance the closed-loop bandwidth, this paper combines the simultaneous IRC damping and tracking control design by mimicking the Butterworth poleplacement pattern with the proposed active resonance shifting loop. Brief introduction and simulation results of this technique have been presented by us at a conference [34]. Different from this, the limitations of this scheme encountered during real-time implementation are detailed, and the control parameters are analytically derived using Butterworth-pattern design method. Meanwhile, an empirical method is employed to fine tune the tracking gain to handle the influence from the inherent system delay, resulting in a flat response. Additionally, comparative experiments with the traditional IRC (without the proposed active control method) are conducted on one axis of a two-dimensional nanopositioner, and the experimentally recorded positioning results are quantified, demonstrating the efficacy of this method in a practical setting.

The remainder of this paper is organized as follows: Section 2 describes the proposed active shifting of the resonant frequency, followed by the simultaneous Butterworth-pattern mimicking design of the IRC and integer tracking control; Section 3 describes the experimental setup and the controller implementation; Section 4 reports and quantifies the experimental results, in terms of bandwidth and tracking performance to show the advantages of the proposed control scheme; and Section 5 concludes this work.

\section{Control design}

As mentioned earlier, the proposed control scheme is composed of three separate loops that work in tandem:

i) Inner loop: Unity feedback control loop with feedforward gain to actively increase the resonance frequency of the system.

ii) Intermediate loop: Damping control loop employing the IRC scheme.

iii) Outer loop: Tracking control loop employing an integral controller.

In the following subsections, the basic principle of operation, the individual structure and their simultaneous Butterworth-pattern mimicking pole-placement design technique are described in detail. As with all such control development, this one also begins by first modeling the dynamics of a typical nanopositioner's axis as a simple second-order transfer function that accurately captures its dominant resonant dynamics. Thus, the dynamics of one axis $G$ can be modeled by a transfer function given by

$$
G=\frac{\sigma^{2}}{s^{2}+2 \zeta \omega_{\mathrm{p}} s+\omega_{\mathrm{p}}^{2}},
$$

where $\omega_{\mathrm{p}}$ is the resonant frequency in $\mathrm{rad} / \mathrm{s}, \zeta$ is the damping coefficient and $\sigma^{2}$ is the gain to match with DC gain of system dynamics.

\subsection{Active control of resonant frequency}

It is well-known that for a second-order transfer function given in Eq. (1), a unity gain negative feedback with a feedforward gain, as shown in Fig. 1, is capable of arbitrarily shifting the resonance of the Plant [34]. However, this shift in resonance comes at the cost of a proportional change in system damping ratio. Therefore, as the resonance is shifted to a higher frequency than its nominal value, the resonant peak at the shifted (higher) frequency gets sharper (damping coefficient is lower). This mechanism can be explained as follows.

Consider the closed-loop arrangement shown in Fig. 1, where the second-order transfer-function ( $G$ as given in Eq. (1)) modeling the nanopositioner axis' dominant, lowly-damped first resonant mode is connected to a gain $K_{\mathrm{s}}$ and a unity-gain negative feedback loop is engaged. 


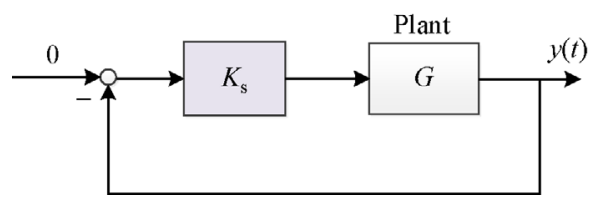

Fig. 1 Block diagram of the inner loop with unity feedback and feedforward gain $\left(K_{\mathrm{s}}\right)$ to actively shift the Plant's $(G)$ resonance. The 'Plant' is the nanopositioner's axis being controlled.

Then, the closed-loop transfer-function of this loop can be resolved as follows:

$$
\begin{aligned}
\hat{G} & =\frac{G K_{\mathrm{s}}}{1+G K_{\mathrm{s}}}=\frac{K_{\mathrm{s}} \sigma^{2}}{s^{2}+2 \zeta \omega_{\mathrm{p}} s+\omega_{\mathrm{p}}^{2}+K_{\mathrm{s}} \sigma^{2}} \\
& =\frac{\hat{\sigma}^{2}}{s^{2}+2 \hat{\zeta} \hat{\omega}_{\mathrm{p}} s+\hat{\omega}_{\mathrm{p}}^{2}},
\end{aligned}
$$

with $\hat{\sigma}^{2}=K_{\mathrm{s}} \sigma^{2}, \hat{\omega}_{\mathrm{p}}^{2}=\omega_{\mathrm{p}}^{2}+K_{\mathrm{s}} \sigma^{2}$, and $\hat{\zeta} \hat{\omega}_{\mathrm{p}}=\zeta \omega_{\mathrm{p}}$. Therefore, in order to increase the resonant frequency $\hat{\omega}_{\mathrm{p}}$, the condition $K_{\mathrm{s}}>0$ has to be satisfied. Comparing Eqs. (1) and (2), it can be found that the original damping ratio and the resulting damping ratio have the following relationship

$$
\frac{\zeta}{\hat{\zeta}}=\frac{\hat{\omega}_{\mathrm{p}}}{\omega_{\mathrm{p}}}
$$

This inverse proportionality between the resonant frequency and damping coefficient shows that an increase in resonant frequency is achieved with a penalty on damping ratio, implying a taller/sharper resonant peak. Though this method allows for arbitrary shifting of the dominant resonant peak, it must be noted that this shift can cause detrimental interference with the higher frequency resonant dynamics. Moreover, extremely lowly-damped resonances reduce phase margins significantly and eliminate any stable closed-loop control implementation. Additionally, it will also be shown in Section 3 that there are hardware-based implementation limitations to the amount of frequency shift that can be imparted to this resonant mode.
2.2 Design of the Butterworth-pattern-based damping and tracking control

Though the sequential design of damping and control loops is prevalent in nanopositioning applications, it was demonstrated recently that simultaneous design/tuning of both damping and tracking control parameters results in maximizing the achievable positioning bandwidth [32]. It was further shown that tuning the damping and tracking controller parameters such that closed-loop poles of the damped and tracked system mimic the Butterworth-filter pattern results in a maximally flat closed-loop response [33].

As the shifting of the dominant resonant mode to a higher frequency comes at the cost of a lower damping coefficient, an aggressive damping control scheme must be adopted to compensate for it. Several prior investigations have reported the superior damping results obtained by employing the IRC scheme. Furthermore, its tuning simplicity and guaranteed closed-loop stability makes it the damping scheme of choice for this resonance-shifted system. Positioning accuracy can be further improved by incorporating suitable tracking controller to work in tandem with the IRC damping scheme. The overall control diagram of the proposed control scheme is shown in Fig. 2, where $d$ is the feedforward term used in the IRC scheme to swap the pole-zero interlacing [29], and $K_{\mathrm{d}}$ and $K_{\mathrm{t}}$ are the integral gains incorporated in the damping and tracking loops, respectively.

Using Eq. (2), the closed-loop transfer function of the system (from reference input $r(t)$ to output $y(t)$ ), whose block diagram representation is given in Fig. 2 can be expressed as $C(s)=N(s) / D(s)$, where the numerator and denominator are

$$
N(s)=K_{\mathrm{d}} K_{\mathrm{t}} K_{\mathrm{s}} \sigma^{2}
$$

and

$$
\begin{aligned}
D(s)= & s^{4}+\left(2 \zeta \omega_{\mathrm{p}}-d K_{\mathrm{d}}\right) s^{3}+\left(\omega_{\mathrm{p}}^{2}+K_{\mathrm{s}} \sigma^{2}-2 \zeta \omega_{\mathrm{p}} d K_{\mathrm{d}}\right) s^{2} \\
& +\left[-\left(\omega_{\mathrm{p}}^{2}+K_{\mathrm{s}} \sigma^{2}\right) d K_{\mathrm{d}}-K_{\mathrm{s}} K_{\mathrm{d}} \sigma^{2}\right] s+K_{\mathrm{d}} K_{\mathrm{t}} K_{\mathrm{s}} \sigma^{2} .
\end{aligned}
$$

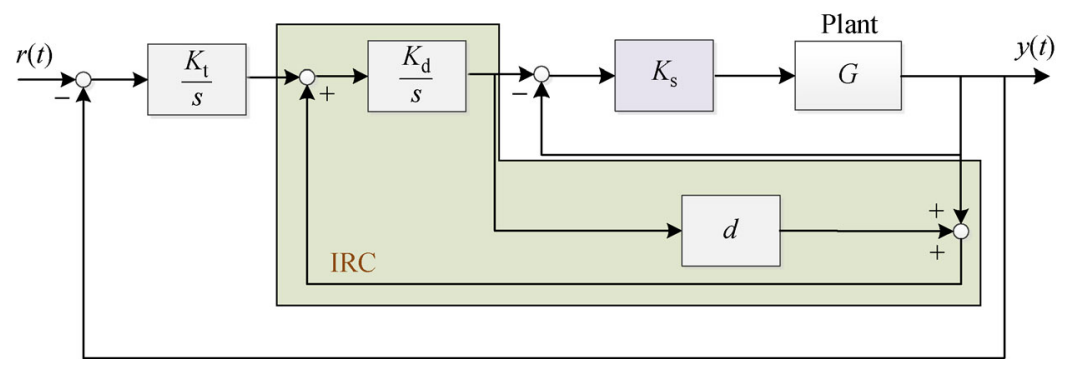

Fig. 2 The overall control diagram with active control of resonance mode. 
It is clear that the closed-loop damped and tracked system is a 4th-order system. In order to enforce a Butterworthmimicking pole placement with the natural frequency of $\omega_{\mathrm{d}}$ and the desired damping ratios of $\gamma_{1}$ and $\gamma_{2}$, the denominator of the closed-loop system $(D(s)$ in Eq. (5)) should be equated to that of a 4th-order Butterworth filter given by

$$
\begin{aligned}
P(s) & =\left(s^{2}+2 \gamma_{1} \omega_{\mathrm{d}} s+\omega_{\mathrm{d}}^{2}\right)\left(s^{2}+2 \gamma_{2} \omega_{\mathrm{d}} s+\omega_{\mathrm{d}}^{2}\right) \\
& =s^{4}+\alpha \omega_{\mathrm{d}} s^{3}+\beta \omega_{\mathrm{d}}^{2} s^{2}+\alpha \omega_{\mathrm{d}}^{3} s+\omega_{\mathrm{d}}^{4},
\end{aligned}
$$

with the intermediate variables $\alpha=2 \gamma_{1}+2 \gamma_{2}$ and $\beta=2+4 \gamma_{1} \gamma_{2}$. Assuming uniform distribution of poles for the Butterworth filter, we can compute $\gamma_{1}=$ $\cos \left( \pm 22.5^{\circ}\right)=0.9239$ and $\gamma_{2}=\cos \left( \pm 67.5^{\circ}\right)=0.3827$. By equating the corresponding coefficients in Eqs. (5) and (6), the following relationships can be obtained:

$$
\begin{gathered}
\alpha \omega_{\mathrm{d}}=2 \zeta \omega_{\mathrm{p}}-d K_{\mathrm{d}}, \\
\beta \omega_{\mathrm{d}}^{2}=\omega_{\mathrm{p}}^{2}+K_{\mathrm{s}} \sigma^{2}-2 \zeta \omega_{\mathrm{p}} d K_{\mathrm{d}}, \\
\alpha \omega_{\mathrm{d}}^{3}=-\left(\omega_{\mathrm{p}}^{2}+K_{\mathrm{s}} \sigma^{2}\right) d K_{\mathrm{d}}-K_{\mathrm{s}} K_{\mathrm{d}} \sigma^{2}, \\
\omega_{\mathrm{d}}^{4}=K_{\mathrm{d}} K_{\mathrm{t}} K_{\mathrm{s}} \sigma^{2} .
\end{gathered}
$$

Combining Eqs. (7) and (8) results in the following equality:

$$
\omega_{\mathrm{d}}=\frac{\zeta \omega_{\mathrm{p}} \alpha}{\beta} \pm \frac{\sqrt{\zeta^{2} \omega_{\mathrm{p}}^{2} \alpha^{2}-\beta\left[4 \zeta^{2} \omega_{\mathrm{p}}^{2} \alpha^{2}-\left(\omega_{\mathrm{p}}^{2}+K_{\mathrm{s}} \sigma^{2}\right)^{2}\right]}}{\beta} .
$$

Similarly, combining Eqs. (7) and (9) results in

$$
K_{\mathrm{d}}=\frac{\alpha \omega_{\mathrm{d}}\left(\omega_{\mathrm{p}}^{2}+K_{\mathrm{s}} \sigma^{2}\right)^{2}}{K_{\mathrm{s}} \sigma^{2}}-\frac{2 \zeta\left(\omega_{\mathrm{p}}^{2}+K_{\mathrm{s}} \sigma^{2}\right)^{3}}{K_{\mathrm{s}} \sigma^{2}}-\frac{\alpha \omega_{\mathrm{d}}^{3}}{K_{\mathrm{s}} \sigma^{2}} .
$$

The parameters $d$ and $K_{\mathrm{t}}$ are recursively determined from Eqs. (7) and (10) by employing the following equalities:

$$
d=\frac{2 \zeta \omega_{\mathrm{p}}-\alpha \omega_{\mathrm{d}}}{K_{\mathrm{d}}}
$$

and

$$
K_{\mathrm{t}}=\frac{\omega_{\mathrm{d}}^{4}}{K_{\mathrm{d}} K_{\mathrm{s}} \sigma^{2}} .
$$

With all the control parameters determined, the proposed active resonance-shifting control scheme can be designed and implemented. The next section presents details of experimental verification and performance evaluation of the closed-loop system controlled by the proposed scheme.

\section{Experimental setup and controller implementation}

In order to evaluate the performance of the proposed active control scheme, the experiments are conducted on a twoaxis piezo-actuated nanopositioner, designed by the EasyLab, University of Nevada, USA [35]. The stage is actuated by two piezoelectric stack actuators, which gives the travel range of $\pm 20 \mu \mathrm{m}$. Two amplifiers (PDL 200 from PiezoDrive, Australia) are employed to provide the highvoltage actuation signals ranging from 0 to $200 \mathrm{~V}$. The high-resolution capacitive sensor (Microsense 6810, with the probe of 2804 and the range of $\pm 50 \mu \mathrm{m}$ ) is used to capture the real-time displacement signals for system modeling and closed-loop control. The control algorithms are implemented using LabVIEW with real-time module, and the signals between the stage and the control system are transferred via the National Instruments card (PCI6621). A general view of the experimental setup is shown in Fig. 3. Since the slow-axis suffers more from the low and lightly-damped resonance, the slow-axis is chosen as the testing axis in this work. The sampling rate of the realtime control module is set as $20 \mathrm{kHz}$.

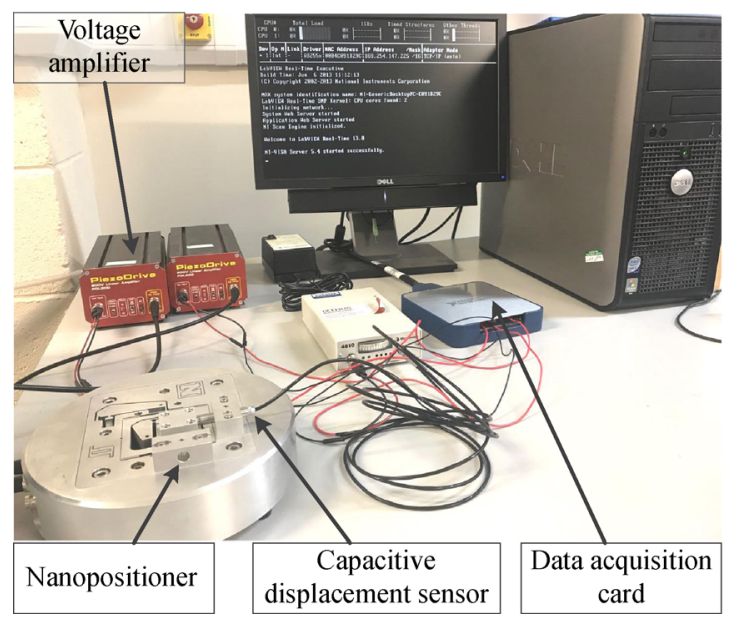

Fig. 3 Experimental setup, including the nanopositioner, the displacement sensor, the voltage amplifier, the data acquisition module and the PC.

To commence with the control design, the linear dynamics of the first resonant mode of the nanopositioner's candidate axis are identified by means of a chirp-based frequency response identification. The input chirp is bandlimited linearly ranging within 10 and $2000 \mathrm{~Hz}$. Fast Fourier transforms of the simultaneously captured input voltage and output displacement signals are computed and combined to generate a frequency response. A least-square method is then employed to obtain a second-order transfer function that accurately captures these first-mode resonant dynamics. The identified transfer 
function is given by

$$
G=\frac{4.58 \times 10^{6}}{s^{2}+73.21 s+1.02 \times 10^{7}} .
$$

Figure 4 shows how accurately the second-order transfer given in Eq. (15) captures the dominant first resonance dynamics. It is also seen that the higher-frequency dynamics occur at frequencies above $1.2 \mathrm{kHz}$. This information will be used as a guideline to limit the amount of shift, the active resonance-shifting loop imparts to the system in order to ensure that the shifted resonance does not interfere with the high-frequency dynamics. It is observed that the first resonant-mode occurs at a frequency of $508 \mathrm{~Hz}$.

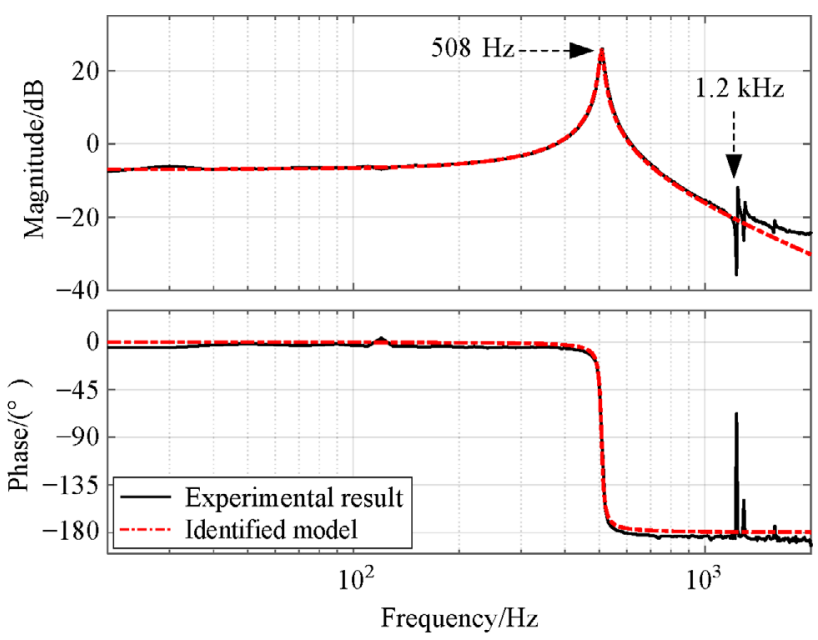

Fig. 4 Plot of the measured (solid black) and modeled (dashed red) frequency responses demonstrating that the model accurately captures the dynamics of the dominant resonant mode which occurs at $508 \mathrm{~Hz}$.

In order to increase the overall closed-loop positioning bandwidth, the first resonant mode is shifted to a higher frequency. However, to ensure that this shifted resonance does not interfere with the unmodeled high-frequency dynamics that occur beyond $1.2 \mathrm{kHz}$, a resonance shift of $400 \mathrm{~Hz}$ was deemed suitable. Thus, the new actively shifted resonance now occurs at $908 \mathrm{~Hz}$ as shown in Fig. 5. Using Eq. (2), the shifting gain $\left(K_{\mathrm{s}}\right)$ required to implement this shift is 4.883 . As expected, Fig. 5 also confirms that this shift in resonance is accompanied by a decrease in the damping coefficient, resulting in a sharper/taller resonant peak.

Using Eqs. (7)-(14), the Butterworth-pattern mimicking damping and tracking control parameters are computed to be:

$$
d=-0.9827, K_{\mathrm{d}}=8209.64, K_{\mathrm{t}}=513.19 .
$$

However, during real-time implementation, these parameters result in a noticeable hump in the (desired) flat-band

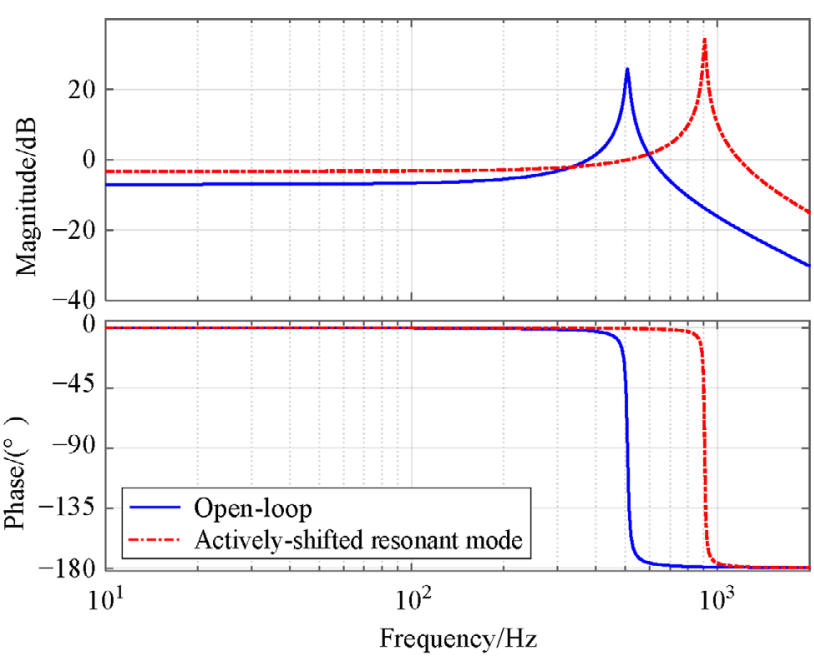

Fig. 5 Nominal open-loop resonant mode (solid blue) at $508 \mathrm{~Hz}$ and actively-shifted resonant mode (dashed red) at $908 \mathrm{~Hz}$. Note that the shift in frequency comes at the cost of a reduction in the damping coefficient (sharper/taller resonant peak).

close-loop response, mainly due to the inherent system delay [35]. To address this issue, the tracking gain $K_{\mathrm{t}}$ is adjusted in experiments via trial-and-error to eliminate this artefact. The tuned $K_{\mathrm{t}}=400$.

To show the advantages the proposed active resonanceshifting control scheme, another set of control gains mimicking the Butterworth-pattern but not incorporating the resonance-shifting loop are determined and implemented (as reported in Ref. [33]). These control parameters are:

$$
d=-0.6490, K_{\mathrm{d}}=6954.48, K_{\mathrm{t}}=297.96 .
$$

Similar to the resonance-shifted case, the tracking gain is adjusted to $K_{\mathrm{t}}=104$ to eliminate the system-delay induced artefact. The design methodology considering the influence of the system delay would be our future focus to further improve the bandwidth as well as the tracking performance. The following section discusses the experimental results obtained.

\section{Experimental results}

This section presents both frequency- and time-domain closed-loop experimental results, clearly demonstrating the efficacy of the proposed control scheme in effectively increasing the achievable positioning bandwidth.

\subsection{Bandwidth test}

The $-3 \mathrm{~dB}$ positioning bandwidth is by far the most common and important performance quantifier for any precise positioning system. Therefore, closed-loop frequency responses were measured using the same technique as described in Section 3 for both the active 
resonance-shifted scheme as well as the standard damping and tracking scheme without the resonance shift. The recorded frequency responses are compared in Fig. 6 for different control techniques. As seen, the nominal damped and tracked closed-loop delivers a $-3 \mathrm{~dB}$ bandwidth of 73 $\mathrm{Hz}$, where the system with the active resonance-shifting scheme delivers a closed-loop bandwidth of $576 \mathrm{~Hz}$, which is not only about 7 times greater than the nominal closedloop bandwidth, but is also greater than the nominal openloop resonant frequency of $508 \mathrm{~Hz}$. To verify how this improvement in bandwidth manifests in time-domain tracking the trajectories, a complete time-domain analysis is performed with the fundamental frequencies of 50 and $100 \mathrm{~Hz}$.

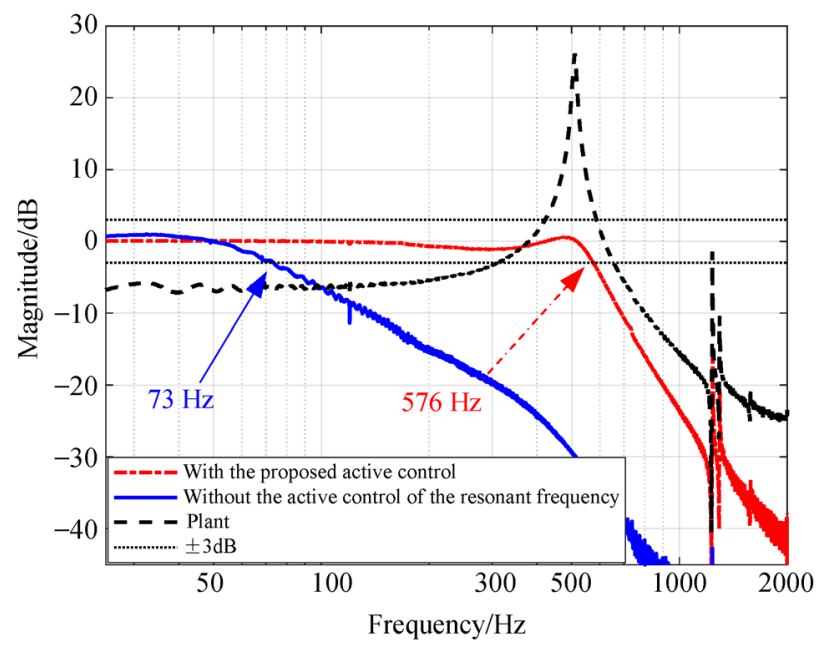

Fig. 6 The magnitude responses of the plant, and the closed-loop systems under the proposed active control and the traditional control without the active control of the resonant frequency.

\subsection{Tracking performance}

For the scanning applications of AFMs, the triangular trajectory is designed as the target trajectories. Therefore, the tracking experiments of the triangular trajectory is conducted on the setup described in the last section. In particular, the delay between the reference trajectory and the actual displacement can be removed via the postimaging process for AFM scanning application, the shifted displacement is deemed as the actual displacement for comparison. Figure 7 shows the results under different control schemes are demonstrated for the tracking frequencies of 50 (Figs. 7(a) and 7(c)) and $100 \mathrm{~Hz}$ (Figs. 7(b) and 7(d)), where the actual displacements in Figs. 7(a) and 7(b) are offset by $1 / 2$ unit to clearly show their difference in time domain. In addition, the tracking results of the system in open-loop are provided as well to show the effectiveness of these two schemes on damping control of the lightly-damped resonance. The tracking errors are compared in the bottom figure for different control schemes. It is obvious in Fig. 7 that the vibrations occur for the system in open-loop and thus cause larger tracking error. In comparison, the problem of vibrations is well addressed by the IRC-based schemes (under the traditional control and the proposed active control). Further, it can be found in this figure that the system under traditional control failed to track the corner of the triangular trajectory due to the lower bandwidth, even for the frequency of $50 \mathrm{~Hz}$. In contrast, under the proposed active control scheme, the tracking performances for triangular trajectories are improved significantly for all the frequencies.

In order to quantitatively evaluate these improvements, the maximum tracking error and the root-mean-square (rms) tracking error for the tracking results are compared in Table 1, which are defined by

$$
\begin{gathered}
e_{\max }=\frac{\max _{t \in(0,2 T]}|r(t)-y(t)|}{\max (r(t))-\min (r(t))}, \\
e_{\text {rms }}=\frac{\sqrt{\frac{1}{2 T} \sum_{t \in(0,2 T]}[r(t)-y(t)]^{2}}}{\max (r(t))-\min (r(t))} .
\end{gathered}
$$

With the improved bandwidth achieved using the proposed active control scheme, the tracking errors are reduced greatly, in which $e_{\max }$ for tracking the triangular trajectory with the fundamental frequency of $100 \mathrm{~Hz}$ is reduced by $87 \%$. Specially, the tracking errors for the triangular trajectory with the frequency of $100 \mathrm{~Hz}$ are obtained as $e_{\max }=4.36 \%$ and $e_{\mathrm{rms}}=1.09 \%$ for the stage with the resonance of $508 \mathrm{~Hz}$. Besides, to quantitatively show how close the actual displacement is to the perfect tracking of the desired trajectories, the percentages of tracking error within a particular error (1\% and 5\%) are additionally presented for these two trajectories, which are compared in Table 1 as well for the system with different control schemes. It can be found that percentage of tracking the $100-\mathrm{Hz}$ triangular trajectory within $5 \%$ error are increased from $58.18 \%$ to $100 \%$ with the proposed active control scheme. This result implies that the prefect tracking of the triangular trajectory within 5\% is achieved, which demonstrates that the proposed control scheme contributes greatly to high-speed and high-precision tracking of nanopositioning stages challenging from a low and lightly damped resonance.

\section{Conclusions}

This paper proposes a novel active control scheme to increase both the resonant frequency and damping ratio for high bandwidth control of the piezo-actuated nanopositioning stage suffering from the low and lightly-damped 
(a)

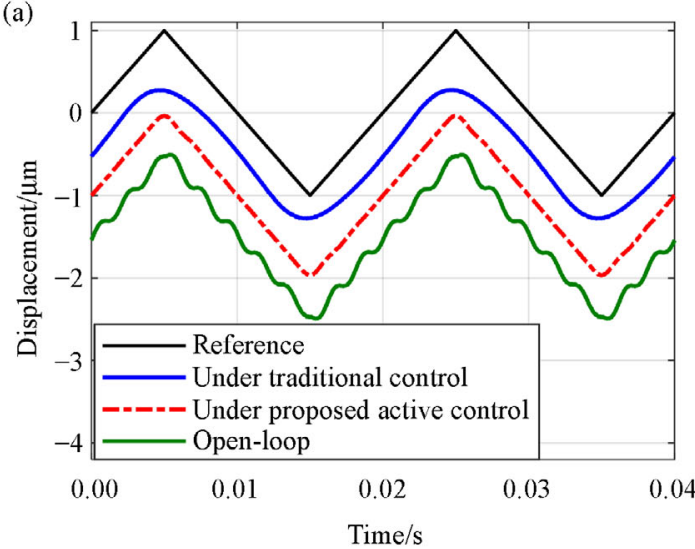

(c)

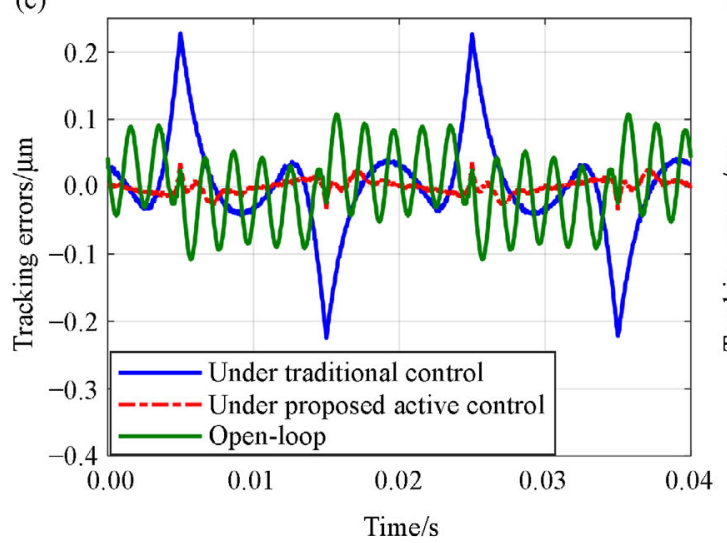

(b)

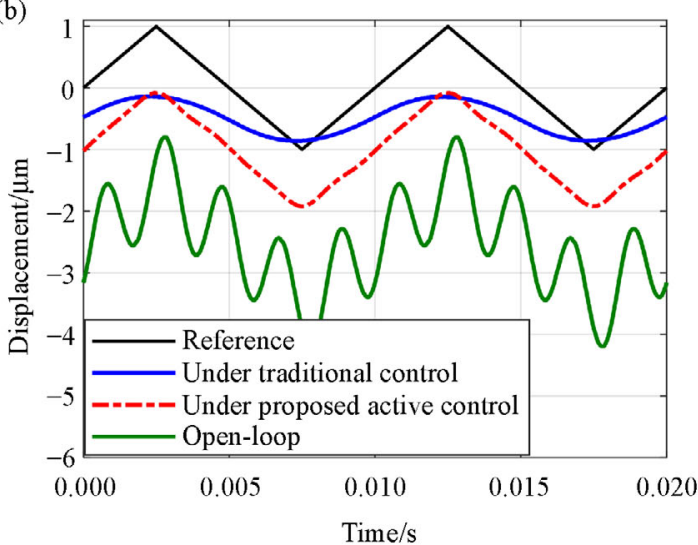

(d)

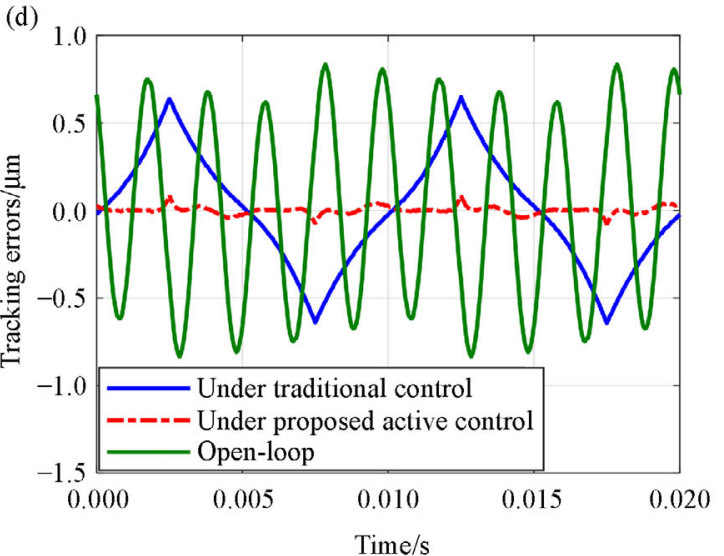

Fig. 7 Tracking results of the system under different control schemes with fundamental frequencies of 50 and $100 \mathrm{~Hz}$. Actual displacement with fundamental frequencies of (a) 50 and (b) $100 \mathrm{~Hz}$. For clarity, the traces are offset by $0.5 \mu \mathrm{m}$. Furthermore, systeminduced phase delay has been eliminated by selecting two full periods of the trajectory for comparison. Tracking errors with fundamental frequencies of (c) 50 and (d) $100 \mathrm{~Hz}$.

Table 1 Indices of the tracking results for the system under different control schemes

\begin{tabular}{|c|c|c|c|c|c|}
\hline Frequency/Hz & Control scheme & $e_{\max } / \%$ & $e_{\mathrm{rms}} / \%$ & $\begin{array}{l}\text { Percentage of tracking } \\
\text { within } 1 \% \text { error } / \%\end{array}$ & $\begin{array}{l}\text { Percentage of tracking } \\
\text { within } 5 \% \text { error } / \%\end{array}$ \\
\hline \multirow[t]{2}{*}{50} & $\begin{array}{l}\text { Without active control of resonant } \\
\text { frequency (traditional) }\end{array}$ & 11.40 & 3.62 & 64.67 & 92.01 \\
\hline & $\begin{array}{l}\text { With active control of resonant } \\
\text { frequency (proposed) }\end{array}$ & 1.83 & 0.50 & 97.00 & 100.00 \\
\hline \multirow[t]{2}{*}{100} & $\begin{array}{l}\text { Without active control of resonant } \\
\text { frequency (traditional) }\end{array}$ & 33.52 & 17.79 & 51.69 & 58.18 \\
\hline & $\begin{array}{l}\text { With active control of resonant } \\
\text { frequency (proposed) }\end{array}$ & 4.36 & 1.09 & 84.89 & 100.00 \\
\hline
\end{tabular}

resonance. Although it is found that the increase of the resonance frequency is at the cost of decreasing the damping ratio, the Butterworth-pattern-based IRC and Integer tracking controller are incorporated to provide the flat response of the overall closed-loop system. The design process is detailed presented. The experiments, including the bandwidth test and trajectories tracking, are conducted on a piezo-actuated nanopositioner. As compared to the scheme without the active control of the resonant frequency, the experimental results show that the bandwidth is improved from 73 to $576 \mathrm{~Hz}$, which confirms the effectiveness of the proposed active control scheme at delivering high-speed scanning.

Acknowledgements This work was supported in part by the National Natural Science Foundation of China (Grant Nos. U2013211 and 51975375), 
the Open Foundation of the State Key Laboratory of Fluid Power and Mechatronic Systems, China (Grant No. GZKF-202003), and the Binks Trust Visiting Research Fellowship (2018), University of Aberdeen, UK, awarded to Dr. Sumeet S. Aphale.

Open Access This article is licensed under a Creative Commons Attribution 4.0 International License, which permits use, sharing, adaptation, distribution and reproduction in any medium or format, as long as you give appropriate credit to the original author(s) and the source, provide a link to the Creative Commons licence, and indicate if changes were made.

The images or other third party material in this article are included in the article's Creative Commons licence, unless indicated otherwise in a credit line to the material. If material is not included in the article's Creative Commons licence and your intended use is not permitted by statutory regulation or exceeds the permitted use, you will need to obtain permission directly from the copyright holder.

To view a copy of this licence, visit http://creativecommons.org/licenses/ by $/ 4.0 \%$.

\section{References}

1. Binnig G, Quate C F, Gerber C. Atomic force microscope. Physical Review Letters, 1986, 56(9): 930-933

2. Dufrêne Y F, Ando T, Garcia R, et al. Imaging modes of atomic force microscopy for application in molecular and cell biology. Nature Nanotechnology, 2017, 12(4): 295-307

3. Shibata T, Iio N, Furukawa H, et al. Nanofabrication technique based on localized photocatalytic reactions using a $\mathrm{TiO}_{2}$-coated atomic force microscopy probe. Applied Physics Letters, 2017, 110(6): 063701

4. Zhang $\mathrm{P}$, Liu $\mathrm{X}$, Liu $\mathrm{P}$, et al. Capturing transient antibody conformations with DNA origami epitopes. Nature Communications, 2020, 11(1): 3114

5. Rana M S, Pota H R, Petersen I R. Improvement in the imaging performance of atomic force microscopy: A survey. IEEE Transactions on Automation Science and Engineering, 2017, 14(2): 12651285

6. Xie H, Wen Y, Shen X, et al. High-speed AFM imaging of nanopositioning stages using $H_{\infty}$ and iterative learning control. IEEE Transactions on Industrial Electronics, 2020, 67(3): 24302439

7. Bhikkaji B, Ratnam M, Fleming A J, et al. High-performance control of piezoelectric tube scanners. IEEE Transactions on Control Systems Technology, 2007, 15(5): 853-866

8. Habibullah H, Pota H R, Petersen I R. A robust control approach for high-speed nanopositioning applications. Sensors and Actuators A: Physical, 2019, 292: 137-148

9. Mahmood I A, Moheimani S O R. Making a commercial atomic force microscope more accurate and faster using positive position feedback control. Review of Scientific Instruments, 2009, 80(6): 063705

10. Raghunvanshi D S, Moore S I, Fleming A J, et al. Electrode configurations for piezoelectric tube actuators with improved scan range and reduced cross-coupling. IEEE/ASME Transactions on Mechatronics, 2020, 25(3): 1479-1486

11. Aphale S S, Bhikkaji B, Moheimani S O R. Minimizing scanning errors in piezoelectric stack-actuated nanopositioning platforms. IEEE Transactions on Nanotechnology, 2008, 7(1): 79-90
12. Gu G Y, Zhu L M, Su C Y, et al. Modeling and control of piezoactuated nanopositioning stages: A survey. IEEE Transactions on Automation Science and Engineering, 2016, 13(1): 313-332

13. Cai K, He X, Tian Y, et al. Design of a XYZ scanner for home-made high-speed atomic force microscopy. Microsystem Technologies, 2018, 24(7): 3123-3132

14. Schitter G, Thurner P J, Hansma P K. Design and input-shaping control of a novel scanner for high-speed atomic force microscopy. Mechatronics, 2008, 18(5-6): 282-288

15. Yang M J, Niu J B, Li C X, et al. High-bandwidth control of nanopositioning stages via an inner-loop delayed position feedback. IEEE Transactions on Automation Science and Engineering, 2015, 12(4): 1357-1368

16. He W, Gao H, Zhou C, et al. Reinforcement learning control of a flexible two-link manipulator: An experimental investigation. IEEE Transactions on Systems, Man, and Cybernetics: Systems, 2020 (in press)

17. He W, Xue C, Yu X, et al. Admittance-based controller design for physical human-robot interaction in the constrained task space. IEEE Transactions on Automation Science and Engineering, 2020, 17(4): 1937-1949

18. Kong L, He W, Yang C, et al. Adaptive fuzzy control for coordinated multiple robots with constraint using impedance learning. IEEE Transactions on Cybernetics, 2019, 49(8): 30523063

19. Rana M S, Pota H R, Petersen I R. A survey of methods used to control piezoelectric tube scanners in high-speed AFM imaging. Asian Journal of Control, 2018, 20(4): 1379-1399

20. Li Y, Xu Q. A novel piezoactuated XY stage with parallel, decoupled, and stacked flexure structure for micro-/nanopositioning. IEEE Transactions on Industrial Electronics, 2011, 58(8): 36013615

21. Yong Y K, Moheimani S O R, Kenton B J, et al. Invited review article: High-speed flexure-guided nanopositioning: Mechanical design and control issues. Review of Scientific Instruments, 2012, 83(12): 121101

22. Vorbringer-Dorozhovets N, Hausotte T, Manske E, et al. Novel control scheme for a high-speed metrological scanning probe microscope. Measurement Science and Technology, 2011, 22(9): 094012

23. Chen G, Liu P, Ding H. Structural parameter design method for a fast-steering mirror based on a closed-loop bandwidth. Frontiers of Mechanical Engineering, 2020, 15(1): 55-65

24. Fanson J L, Caughey T K. Positive position feedback control for large space structures. AIAA Journal, 1990, 28(4): 717-724

25. Bhikkaji B, Ratnam M, Moheimani S O R. PVPF control of piezoelectric tube scanners. Sensors and Actuators A: Physical, 2007, 135(2): 700-712

26. Li L, Li C X, Gu G, et al. Positive acceleration, velocity and position feedback based damping control approach for piezo-actuated nanopositioning stages. Mechatronics, 2017, 47: 97-104

27. Namavar M, Fleming A J, Aleyaasin M, et al. An analytical approach to integral resonant control of second-order systems. IEEE/ASME Transactions on Mechatronics, 2014, 19(2): 651-659

28. Bhikkaji B, Moheimani S O. Integral resonant control of a piezoelectric tube actuator for fast nanoscale positioning. IEEE/ 
ASME Transactions on Mechatronics, 2008, 13(5): 530-537

29. Aphale S S, Fleming A J, Moheimani S O R. Integral resonant control of collocated smart structures. Smart Materials and Structures, 2007, 16(2): 439-446

30. Fleming A J. Nanopositioning system with force feedback for highperformance tracking and vibration control. IEEE/ASME Transactions on Mechatronics, 2010, 15(3): 433-447

31. Habibullah H, Pota H R, Petersen I R, et al. Tracking of triangular reference signals using LQG controllers for lateral positioning of an AFM scanner stage. IEEE/ASME Transactions on Mechatronics, 2014, 19(4): 1105-1114

32. Russell D, Fleming A J, Aphale S S. Simultaneous optimization of damping and tracking controller parameters via selective pole placement for enhanced positioning bandwidth of nanopositioners.
Journal of Dynamic Systems, Measurement, and Control, 2015, 137(10): 101004

33. Russell D, San-Millan A, Feliu V, et al. Butterworth pattern-based simultaneous damping and tracking controller designs for nanopositioning systems. Frontiers in Mechanical Engineering, 2016, 2: 110

34. Aphale S S, Namavar M, Fleming A J. Resonance-shifting integral resonant control for high-speed nanopositioning. In: Proceedings of Annual American Control Conference. Milwaukee: IEEE, 2018, 6006-6011

35. San-Millan A, Russell D, Feliu V, et al. A modified positive velocity and position feedback scheme with delay compensation for improved nanopositioning performance. Smart Materials and Structures, 2015, 24(7): 075021 